\title{
A CHARACTERIZATION OF MANIFOLDS
}

\author{
BY
}

LOUIS F. MCAULEY

ABSTRACT. The purpose of this paper is (1) to give a proof of one general theorem characterizing certain manifolds and (2) to illustrate a technique which should be useful in proving various theorems analogous to the one proved here.

THEOREM. Suppose that $f: X \Rightarrow[0,1]$, where $X$ is a compactum, and that $f$ has the properties:

(1) for $0 \leqslant x<1 / 2, f^{-1}(x)=S^{n} \cong M_{0}$,

(2) $f^{-1}(1 / 2) \cong S^{n}$ with a tame (or flat) $k$-sphere $S^{k}$ shrunk to a point,

(3) for $1 / 2<x \leqslant 1, f^{-1}(x) \cong$ a compact connected n-manifold $M_{1} \cong S^{n-(k+1)} \times S^{k+1}$ (a spherical modification of $M_{0}$ of type $k$ ), and

(4) there is a continuum $C$ in $X$ such that (letting $C_{x}=f^{-1}(x) \cap C$ )

(a) $0 \leqslant x<1 / 2, C_{x} \cong s^{k}$, (b) $C_{1 / 2}=\{p\}$ a point, (c) for $1 / 2<x \leqslant 1$, $C_{x} \cong S^{n-(k+1)}$, and (d) each of $f|(X-C), f| f^{-1}[0,1 / 2)$, and $f \mid f^{-1}(1 / 2,1]$ is completely regular.

Then $X$ is homeomorphic to a differentiable $(n+1)$-manifold $M$ whose boundary is the disjoint union of $\bar{M}_{0}$ and $\bar{M}_{1}$ where $M_{i}=\bar{M}_{i}, i=0,1$.

1. Introduction. There are a number of interesting theorems in differential topology that characterize spheres, but their proofs use "smoothness" of both the manifold and the mapping. For example, the theorem of Reeb [15] and Milnor [10], later generalized by Milnor [11] and Rosen [16], is such a characterization.

THeOREM 1 (REeb-MiLNOR-Rosen). Suppose that $M$ is smooth $\left(C^{\infty}\right)$ compact manifold and that $f$ is a smooth real-valued function on $M$ with exactly two critical points (degenerate or not). Then $M$ is homeomorphic to a sphere.

This theorem has a topological version which we gave in [8]. It is as follows.

Theorem 2 (MCAuley). Suppose that $M$ is a continuum (compact connected metric space) and that $f: M \Rightarrow I=[0,1]$ is a (continuous) mapping.

Received by the editors January 19, 1973 and, in revised form, January 21, 1974.

AMS (MOS) subject classifications (1970). Primary 54B15, 54C10, 54C30, 54C35, 54C65, 57A15; Secondary 55F05, 55F55, 55 F65, 58D99. 
Furthermore, $f^{-1}(0)=a$ (point), $f^{-1}(1)=b$ (point), $f(M-\{a, b\})$ is completely regular, and $f^{-1}(x)$ is homeomorphic to an $n$-sphere $S^{n}$ for each $x \in(0,1)$. Then $M$ is homeomorphic to $S^{n+1}$.

The condition that $f^{-1}(x)$ be an $n$-sphere $S^{n}$ is quite natural in view of the following theorem from differential topology.

THEOREM 3. If $f: M \rightarrow N$ is a smooth mapping between smooth manifolds of dimensions $m$ and $n$, respectively, where $m \geqslant n$ and if $y \in N$ is a regular value, then the set $f^{-1}(y) \subseteq M$ is a smooth manifold of dimension $m-n$.

One wonders just what are the topological properties of differential mappings? Also, under what reasonable (topological) conditions is a mapping differential? In the case of nonconstant analytic mappings from the complex plane to the complex plane, the properties of openness and lightness actually characterize them. Whyburn [21] and Stoilow [18] have shown that if $f: M^{2} \Rightarrow N^{2}$ is a light open mapping between 2 -manifolds, then $f$ is topologically equivalent to an analytic mapping. Several researchers, Church in particular, have made considerable progress in obtaining topological properties of differentiable mappings. For references and results, see [2].

At the topology conference held at the University of Oklahoma, March, 1972, I gave a talk containing outlines of proofs of theorems for which Theorems 2 and 4 (below) are special cases. The manuscript for that talk has appeared in the Proceedings, Topology Conference, University of Oklahoma, 1972.

THEOREM 4 (MCAULEY). Suppose $M$ is a continuum and that $f: M \Rightarrow[0,1]$ is a mapping such that (1) $f^{-1}(0)=a$ (point), (2) $f^{-1}(1)=b$ (point), (3) $f^{-1}(1 / 4)=f^{-1}(3 / 4)=a$ figure eight (two circles with exactly one common point), (4) for $0<x<1 / 4$ or $3 / 4<x<1, f^{-1}(x) \cong a$ circle, (5) for $1 / 4<x<3 / 4$, $f^{-1}(x) \cong a$ pair of disjoint circles, and (6) for $0<x<1$, there is a "triangulation" of $f^{-1}(x)$ which contains exactly four 1-simplexes (simple arcs) and $f$ is completely regular with respect to the collection of all 1-simplexes. Then $M \cong$ torus or Klein bottle.

The purpose of this paper is to give a proof of one general theorem characterizing certain manifolds. Perhaps a more important objective is our illustration of the methods of proof which should be useful in proving various theorems of this kind.

TheOREM 5. Suppose that $X$ is a compact metric space and that $f: X \Rightarrow$ $[0,1]$ has the following properties: 
(1) for $0 \leqslant x<1 / 2, f^{-1}(x)=S^{n} \cong M_{0}$,

(2) $f^{-1}(1 / 2)$ is homeomorphic to $S^{n}$ with a tame or flat $k$-sphere $S^{k}$ shrunk to a point,

(3) for $1 / 2<x \leqslant 1, f^{-1}(x) \cong$ a compact connected n-manifold $M_{1} \cong$ $S^{n-(k+1)} \times S^{k+1}$ which is a spherical modification of $M_{0}$ of type $k$ (a regular neighborhood of $S^{k} \subset M_{0}$, i.e., $S^{k} \times I^{n-k}$, is replaced by $\left.S^{n-(k+1)} \times I^{k+1}\right)$, and

(4) there is a continuum $C$ in $X$ such that (letting $C_{x}=f^{-1}(x) \cap C$ ) (a) for $0 \leqslant x<1 / 2, C_{x} \cong S^{k}$, (b) $C_{1 / 2}=p$, a point-the topological critical point of $f$, (c) for $1 / 2<x \leqslant 1, C_{x} \cong S^{n-(k+1)}$, and (d) each of $f \mid(X-C), f f^{-1}[0,1 / 2)$, and $f \mid f^{-1}(1 / 2,1]$ is completely regular.

Then $X$ is homeomorphic to a differentiable $(n+1)$-manifold $M$ whose boundary is the disjoint union of $\bar{M}_{0}$ and $\bar{M}_{1}$ where $M_{i} \cong \bar{M}_{i}, i=0,1$.

PRoof. Let $\bar{M}_{0}$ and $\bar{M}_{1}$ be differentiable manifolds homeomorphic to $M_{0}$ and $M_{1}$, respectively. There is a differentiable manifold $M$ whose boundary is the disjoint union of $\bar{M}_{0}$ and $\bar{M}_{1}$ and a differentiable function $g$ on $M$ equal to 0 on $\bar{M}_{0}$, equal to 1 on $\bar{M}_{1}$, and otherwise having values between 0 and 1 and having exactly one nondegenerate critical point $q$ (with critical value $1 / 2$, say) with type number $k+1$ [24]. Now, for $0 \leqslant x<1 / 2, g^{-1}(x) \cong S^{n} \cong M_{0} \cong$ $\bar{M}_{0}, g^{-1}(1 / 2) \cong S^{n}$ with a $k$-sphere shrunk to a point, and for $1 / 2<x \leqslant 1$, $g^{-1}(x) \cong M_{1} \cong \bar{M}_{1}$. Furthermore, there is a "smooth" closed and connected set $Z$ such that (1) for $0 \leqslant x<1 / 2, Z_{x}=Z \cap g^{-1}(x) \cong S^{k}$, (2) $g^{-1}(1 / 2) \cap Z=$ $q$, the critical point of $g$, (3) for $1 / 2<x \leqslant 1, Z_{x}=Z \cap g^{-1}(x) \cong S^{n-(k+1)}$, and (4) $Z$ is "canonical" in the sense of Wallace [24, p. 88]. Consider the trajectories to the level sets of $g$. The trajectories starting at points of $Z_{0} \cong S^{k}$ all end at $q$. As we move through the levels of $g$ from $\bar{M}_{0}$ to $\bar{M}_{1}$, the $Z_{x} \cong S^{k}$ shrink to $q$ along the orthogonal trajectories. As we continue above the critical level, $g^{-1}(1 / 2), Z_{x} \cong S^{n-(k+1)}$ grows along the orthogonal trajectories from $q$ to $Z_{1} \subset \bar{M}_{1}$. Thus, in this sense, $Z$ is "canonical".

Clearly, $Z$ is homeomorphic to $C$ (in the hypothesis) and $M$ is homeomorphic to $P=\left(S^{n} \times[0,1 / 2]\right) \cup\left(\left(S^{n-(k+1)} \times S^{k+1}\right) \times[1 / 2,1]\right)$ where $\left(S^{n}, 1 / 2\right)$ and $\left(S^{n-(k+1)} \times S^{k+1}, 1 / 2\right)$ are sewed together in the obvious manner (indicated below). Thus, there is (I) a tame $k$-sphere $S^{k}$ in $S^{n}$, (II) a tame $n-(k+1)$ sphere $S^{n-(k+1)}$ in $\left(S^{n-(k+1)} \times S^{k+1}\right)$, and (III) a continuous mapping $m$ : $P \Rightarrow M$ such that (1) $m \mid\left(S^{n}, x\right), 0 \leqslant x<1 / 2$, is a homeomorphism taking $\left(S^{n}, x\right)$ onto $g^{-1}(x),(2) m \mid\left(S^{n-(k+1)}, x\right), 1 / 2<x \leqslant 1$, is a homeomorphism taking $\left(S^{n-(k+1)}, x\right)$ onto $g^{-1}(x)$, and (3) each of $m \mid\left(S^{n}, 1 / 2\right)$ and $m \mid\left(S^{n-(k+1)} \times\right.$ $\left.S^{k+1}, 1 / 2\right)$ is a homeomorphism off $\left(S^{k}, 1 / 2\right)$ and $\left(S^{n-(k+1)}, 1 / 2\right)$, respectively, 
which takes $\left(S^{n}-S^{k}\right)$ and $\left(S^{n-(k+1)} \times S^{k+1}-S^{n-(k+1)}\right)$ onto $g^{-1}(1 / 2)-$ $\{q\}$ and takes $\left(S^{k}, 1 / 2\right)$ and $\left(S^{n-(k+1)}, 1 / 2\right)$ onto $q$. In the following, it is more convenient to work with $P$ than with $M$.

Let $h_{1}$ denote a mapping of $S^{k} \times[0,1 / 2]$ into $f^{-1}[0,1 / 2]$ (actually, onto $\left.f^{-1}[0,1 / 2] \cap C\right)$ such that $h_{1}$ takes $\left(S^{k}, t\right)$ homeomorphically onto $C_{t}=$ $f^{-1}(t) \cap C$ for $0 \leqslant t<1 / 2$ and takes $\left(S^{k}, 1 / 2\right)$ onto $f^{-1}(1 / 2) \cap C=p$. Similarly, let $h_{2}: S^{n-(k+1)} \times[1 / 2,1] \rightarrow f^{-1}[1 / 2,1]$ take $\left(S^{n-(k+1)}, t\right)$ homeomorphically onto $C_{t}=f^{-1}(t) \cap C$ for $1 / 2<t \leqslant 1$ and takes $\left(S^{n-(k+1)}, 1 / 2\right)$ onto $p=C_{1 / 2}=$ $f^{-1}\left(\frac{1}{2}\right) \cap C$.

For $0 \leqslant t<1 / 2$, let $K_{t}$ be the space of all homeomorphisms of $S^{n}$ onto $f^{-1}(t)$ taking $x \in S^{k}$ onto $h_{1}(x, t)$. Similarly, let $K_{t}$ be the space of all homeomorphisms of $S^{n-(k+1)} \times S^{k+1}$ onto $f^{-1}(t)$ taking $x \in S^{n-(k+1)}$ onto $h_{2}(x, t)$ for $1 / 2<t \leqslant 1$.

Let $K_{1 / 2}^{0}$ be the space of all mappings $w$ of $S^{n}$ onto $f^{-1}(1 / 2)$ taking $x \in S^{k}$ to $h_{1}(x, 1 / 2)=p$ such that $w \mid\left(s^{n}-S^{k}\right)$ is a homeomorphism. Similarly, let $K_{1 / 2}^{1}$ be the space of mappings $w$ of $S^{n-(k+1)} \times S^{k+1}$ onto $f^{-1}(1 / 2)$ taking $x \in S^{n-(k+1)}$ to $h_{2}(x, 1 / 2)=p$ such that $w \mid\left\{S^{n-(k+1)} \times S^{k+1}-S^{n-(k+1)}\right\}$ is a homeomorphism.

We shall consider the collection $L_{0}$ of all $K_{t}, 0 \leqslant t<1 / 2$ plus $K_{1 / 2}^{0}$ and the collection $L_{1}$ of all $K_{t}, 1 / 2<t \leqslant 1$ plus $K_{1 / 2}^{1}$. Now, $L_{i}^{*}$ will denote the union of the elements of $L_{i}$. Next, we define a metric for $L_{i}^{*}$. If $m \in L_{i}^{*}$, let $\hat{m}$ denote the graph of $m$ in $P \times X$. Thus, for each pair $m, n \in L_{i}^{*}$ where $m \in K_{a}$ and $n \in K_{b}$, let $D(m, n)=H(\hat{m}, \hat{n})$ where $H$ denotes the Hausdorff metric on the space of all closed subsets of $P \cap X$. Now, $\left(L_{i}^{*}, D\right)$ is a topologically complete metric space. For a proof, see an argument in [7, Theorem 1] for an analogous result. We let $\rho$ denote a complete metric for $L_{i}^{*}$.

LEMma 1. Each $K_{t}$ and $K_{1 / 2}^{i}, i=0,1$, is $L C^{0}$ (in the homotopy sense). Indeed, each is locally contractible.

Proof. For $0 \leqslant t<1 / 2$, it should be clear that $K_{t}$ is homeomorphic to the space of all homeomorphisms of $S^{n}$ onto itself with a tame (or flat) $k$-sphere $S^{k}$ fixed. Thus, by [4], it follows that $K_{t}$ is locally contractible. Similarly, for $1 / 2<t \leqslant 1, K_{t}$ is locally contractible. Now, $K_{1 / 2}^{i}$ is the space of all homeomorphisms of a compact polyhedron $T$ onto itself keeping a point $s$ fixed where $T$ is the result of shrinking $S^{k}$ (or $S^{n-(k+1)}$ ) in $S^{n}$ (or $S^{n-(k+1)} \times S^{k+1}$ ) to a point. It follows from [22] that $K_{1 / 2}^{i}$ is locally contractible.

Lemma 2. The collections $L_{i}, i=0,1$, are equi-LC $C^{n}$.

Proof. Each $L_{i}^{*}$ is a complete metric space with metric $\rho$. Note that 
$f \mid f^{-1}[0,1 / 2)$ and $f \mid f^{-1}(1 / 2,1]$ are completely regular in the sense of Dyer and Hamstrom [3]. It follows by an argument analogous to that given in [3] that the collection of all $K_{t}$ is equi- $L C^{n}$ for each $n$. To show that $L_{0}$ is equi- $L C^{n}$, we need only consider $\epsilon>0$ and $g \in K_{1 / 2}^{0}$.

Since $K_{1 / 2}^{0}$ is $L C^{n}$, there is a $\delta_{1}>0$ such that each mapping $r: S^{k} \rightarrow$ $K_{1 / 2}^{0} \cap N_{\delta_{1}}(g)$, for $0 \leqslant k \leqslant n$, can be extended to a mapping $R: I^{k+1} \rightarrow$ $K_{1 / 2}^{0} \cap N_{\epsilon / 2}(g)$. Since $f \mid(X-C)$ is completely regular, there is $\alpha>0$ such that if $1 / 2-b<\alpha, b \in[0,1 / 2]$, there is a mapping $m: f^{-1}(b) \Rightarrow f^{-1}(1 / 2)$ such that $m\left(C_{b}\right)=C_{1 / 2}, m \mid\left(f^{-1}(b)-C_{b}\right)$ is a homeomorphism, and $m$ moves no point as much as $\delta_{1} / 2$.

Choose $\delta, 0<\delta<\min \left(\delta_{1} / 2,1 / 2\right)$ such that if $K_{b} \cap N_{\delta}(g) \neq \varnothing$, then $1 / 2-b<\alpha$. Now, let $\phi: S^{k} \rightarrow K_{b} \cap N_{\delta}(g)$. We wish to show that $\phi$ can be extended to $\phi: I^{k+1} \rightarrow K_{b} \cap N_{\epsilon}(g)$. Let $c=1 / 2$. We can define a 1-1 mapping $H_{b c}: K_{b} \rightarrow K_{c}$ as follows: For $e \in K_{b}$, let $H_{b c}(e)=m e \in K_{c}$. Clearly, $H_{b c} \mid\left(K_{b} \cap N_{\delta}(g)\right)$ maps $K_{b} \cap N_{\delta}(g)$ into $K_{c} \cap N_{\delta_{1}}(g)$. In fact, $H_{b c}$ maps $K_{b}$ onto $K_{c}$. Furthermore, $r=\left[H_{b c} \mid \phi\left(S^{k}\right)\right] \phi$ maps $S^{k}$ into $K_{c} \cap N_{\delta_{1}}(g)$ and can be extended to a mapping $R: I^{k+1} \rightarrow K_{c} \cap N_{\epsilon / 2}(g)$ such that for each $p \in I^{k+1}$, $R(p) \in H_{b c}\left(K_{b}\right) \subset K_{c}$ since $H_{b c}\left(K_{b}\right)$ is $L C^{n}$. Now, define $H_{c b}: H_{b c}\left(K_{b}\right) \rightarrow K_{b}$ as $H_{c b}(m e)=e$. Clearly, $H_{c b}$ is the inverse of $H_{b c}$ and $H_{b c}$ is a homeomorphism. Now, $\Phi=\left[H_{c b} \mid H_{b c}\left(K_{b}\right) \cap N_{\epsilon / 2}(g)\right] R$ maps $I^{k+1}$ into $K_{b} \cap N(g)$ and agrees with $\phi$ on $S^{k}$ the boundary of $I^{k+1}$. Thus, $L_{0}$ is equi-LC $C^{n}$. Similarly, it follows that $L_{1}$ is equi- $L C^{n}$.

Lemma 3. The collections $L_{i}$ are lower semicontinuous (lsc) in the sense that if $\left\{x_{i}\right\} \rightarrow x$ in $[0,1 / 2]$ or $[1 / 2,1]$, then $K_{x}$ is in the closure of $U K_{x_{i}}$.

A proof follows easily from the fact that each of $f\left|f^{-1}[0,1 / 2), f\right| f^{-1}(1 / 2,1]$, and $f \mid(X-C)$ is completely regular.

Next, let $F: L_{0}^{*} \Rightarrow[0,1 / 2]$ be the function defined by $F(k)=x$ iff $k \in K_{x}$. Thus, the collection of point inverses under $F$ is the collection $L_{0}$ which is 1 sc and equi- $L C^{n}$. Also, $L_{0}^{*}$ is a complete metric space. Given $x \in[0,1 / 2]$, let $\phi(x) \in K_{x}$. By Michael's section theorem [9], there is an open set $U$ of $[0,1 / 2]$ with $x \in U$ and a continuous extension of $\phi$ to $U$ (denote it by $\Phi$ ) with the property that $\Phi(u) \in K_{u}$ for each $u \in U$. Clearly, $[0,1 / 2]$ is covered by a finite number of closed intervals $\left[a_{i}, b_{i}\right]$ where $a_{0}=0<b_{0}=a_{1}<b_{1}=a_{2}<b_{2} \ldots$ $<b_{t}=1 / 2$ with mappings $m_{i}: S^{n} \times\left[a_{i}, b_{i}\right] \Rightarrow f^{-1}\left[a_{i}, b_{i}\right]$ where $m_{i}$ is a homeomorphism for $i=1,2, \ldots, t-1$ and $m_{t}$ is a homeomorphism off $\left(S^{k}, 1 / 2\right)$ and takes $\left(S^{k}, 1 / 2\right)$ to $p$. Next, we sew the pieces together in the obvious way. Identify $h_{i}\left(x, a_{i}\right)$ with $h_{i+1}\left(x, a_{i}\right)$ for $i=0,1, \ldots, t-1$. We obtain a mapping 
$H_{0}: S^{n} \times[0,1 / 2] \Rightarrow f^{-1}[0,1 / 2]$ which is a homeomorphism except on $\left(S^{k}, 1 / 2\right)$. In a similar way, we obtain a mapping $H_{1}:\left(S^{n-(k+1)} \times S^{k+1}\right) \times[1 / 2,1] \Rightarrow$ $f^{-1}[1 / 2,1]$ which is a homeomorphism except on $\left(S^{n-(k+1)}, 1 / 2\right)$ which maps to $p$. We sew these together to obtain a mapping $H: P \Rightarrow f^{-1}[0,1]=X$ (recalling that $\left.P=\left(S^{n} \times[0,1 / 2]\right) \cup\left(\left(S^{n-(k+1)} \times S^{k-1}\right) \times[1 / 2,1]\right)\right)$ such that $h=$ $H m^{-1}: M \Rightarrow X$ is a homeomorphism (again, recalling that $m: P \Rightarrow M$ has certain properties). Consequently, $X$ is homeomorphic to the differentiable $(n+1)$-manifold $M$. Theorem 5 is proved.

\section{BIBLIOGRAPHY}

1. A. V. Černavskii, Local contractibility of the homeomorphism group of a manifold, Dokl. Akad. Nauk SSSR 182 (1968), 510-513 = Soviet Math. Dokl. 9 (1968), 1171 1174. MR 38 \#5241.

2. P. T. Church, Differentiable monotone mappings and open mappings, Proc. First Conf. on Monotone Mappings and Open Mappings (SUNY at Binghamton, Binghamton, N. Y., 1970), State Univ. of New York at Binghamton, Binghamton, N. Y., 1971, pp. 145-183. MR 43 \#6938.

3. E. Dyer and M.-E. Hamstrom, Completely regular mappings, Fund. Math. 45 (1958), 103-118. MR 19, 1187.

4. R. D. Edwards and R. C. Kirby, Deformations and spaces of imbeddings, Ann. of Math. (2) 93 (1971), 63-88. MR 44 \#1032.

5. R. H. Fox, Covering spaces with singularities, Algebraic Geometry and Topology. A Sympos. in Honor of S. Lefschetz, Princeton Univ. Press, Princeton, N. J., 1957, pp. 243-257. MR 23 \#A629.

6. L. F. McAuley, Some upper semi-continuous decompositions of $E^{3}$ into $E^{3}$, Ann. of Math (2) 73 (1961), 437-457. MR 23 \#A3554.

7. The existence of a complete metric for a special mapping space and some consequences, Topology Seminar (Wisconsin, 1965), Ann. of Math. Studies, no. 60, Princeton Univ. Press, Princeton, N. J., 1966, pp. 135-139. MR 37 \#6903.

8. —_, A topological Reeb-Milnor-Rosen theorem, Bull. Amer. Math. Soc. 78 (1972), 82-84. MR $44 \# 4728$.

9. E. A. Michael, Continuous selections. I, II, III, Ann. of Math. (2) 63 (1956), 361-382; (2) 64 (1956), 562-580; (2) 65 (1957), 357-390. MR 17, 990; 18, 325; 750.

10. J. W. Milnor, On manifolds homeomorphic to the 7-sphere, Ann. of Math. (2) 64 (1956), 399-405. MR 18, 498.

11. - Sommes de variétés différentiables et structures différentiables der sphères, Bull. Soc. France 87 (1959), 439-444. MR 22 \#8518.

12. Morse theory, Ann. of Math. Studies, no. 51, Princeton Univ. Press, Princeton, N. J., 1963. MR 29 \#634.

13. D. Montgomery and H. Samelson, Fiberings with singularities, Duke Math. J. 13 (1946), 51-56. MR 7, 471.

14. W. L. Reddy, Montgomery-Samelson coverings on manifolds, Proc. First Conf. on Monotone Mappings and Open Mappings (SUNY at Binghamton, Binghamton, N. Y., 1970), State Univ. of New York at Binghamton, Binghamton, N. Y., 1971, pp. 192-198. MR 44 \#1002.

15. G. Reeb, Sur certaines properiétés topologiques des variétés feuilletées, Actualités Sci. Indust., no. 1183, Hermann, Paris, 1952, pp. 91-154. MR 14, 1113.

16. R. H. Rosen, $A$ weak form of the star conjecture for manifolds, Notices Amer. Math. Soc. 7 (1960), 380. Abstract \#570-28.

17. Melvin C. Thornton, Singularly fibered manifolds, Illinois Math. J. 11 (1967), 189-201. MR 35 \#1031. 
18. S. Stoilow, Leçons sur les principes topologiques de la théorie fonctions analytiques, Gauthier-Villars, Paris, 1938.

19. J. H. C. Whitehead, On simply connected, 4-dimensional polyhedra, Comment. Math. Helv. 22 (1949), 48-92. MR 10, 559.

20. ——, Combinatorial homotopy. I, Bull. Amer. Math. Soc. 55 (1949), 213245. MR 11, 48.

21. G. T. Whyburn, Analytic topology, Amer. Math. Soc. Colloq. Publ., vol. 28, Amer. Math. Soc., Providence, R. I., 1942. MR 4, 86.

22. L. C. Siebenmann, Deformation of homeomorphisms on stratified sets. I, II, Comment. Math. Helv. 47 (1972), 123-163. MR 47 \#7752.

23. G. T. Whyburn, Topological analysis, 2nd rev. ed., Princeton Math. Ser., no. 23, Princeton Univ. Press, Princeton, N. J., 1964. MR 29 \#2758.

24. A. H. Wallace, Differential topology: First Steps, Benjamin, New York, 1968. MR 36 \#7150.

DEPARTMENT OF MATHEMATICS, STATE UNIVERSITY OF NEW YORK AT BINGHAMTON, BINGHAMTON, NEW YORK 13901 